\title{
ESTABLISHING MAN-MACHINE RATIO USING SIMULATION
}

\author{
Hoay Hoon Ong \\ Bayan Lepas Free Industrial Zone, \\ Phase 3, Halaman Kampung Jawa \\ Intel Technology Sdn. Bhd. \\ Penang, 11900, MALAYSIA
}

\begin{abstract}
Effective use of machines and headcounts is essential in manufacturing for high return of investment from expensive resources in today's competitive business environment. It is thus crucial to establish optimal man-machine ratio to gain high resource utilization and output. In this paper, we discuss the deficiency of traditional static methods and how simulation can be applied to overcome these deficiency in order to establish optimal man-machine ratio. In addition, we discuss using simulation to facilitate decision making on headcount movement from non-constraint tool to constraint tool to boost productivity of constraint tool, and ultimately increase overall factory output.
\end{abstract}

\section{INTRODUCTION}

The common practice in Intel Assembly and Test manufacturing to determine man-machine ratio is to utilize spreadsheet analysis coupled with trial runs to experiment different manning ratio by observing their impact especially machine throughput and resources utilization. Manmachine chart is also used in the industry to determine the optimal quantity of machines a manufacturing specialist (MS) can operate (Institute of Industrial Engineers 2007). However, these approaches have their deficiencies.

The rest of the paper uses the following abbreviations and definition:

MS (Manufacturing Specialists).

Definition 1 Internal work elements refer to work elements performed by MS while machine is running.

Definition 2 External work elements refer to work elements performed by MS while machine is idling.

Definition 3 Machine work elements refer to work elements performed by machine.

Definition 4 Manning ratio 1:x refer to manning ratio where 1 MS operate x number of machines.

Traditional spreadsheet methodology only considers machine and external work elements in manning ratio calculation. By excluding internal work elements, this calcu- lation may accurately account for manning ratio $1: 1$ but may not for other manning ratios. For example, in the event of 1:3 manning ratio, paper work which is classified as internal work elements can only be qualified as "internal" if it was done when all 3 machines are running. However, at 1:3 manning ratio, this does not happen all the time in reality. When some paper work become "external" work elements, machines will be idling waiting for MS resources. The longer the machine idling time, the more the losses is attributed by reduction of machine throughput; one major factor being considered when determining manning ratio.

Man-machine chart, normally generated during labor time study, is a chart of the coordinated synchronous or simultaneous activities of a work system of one or more workers. Figure 1 illustrates an example of man-machine chart. Each machine and/or MS is shown in a separate, parallel column indicating their activities with relative to the rest of the work system. The chart is often used to exemplify the most productive way to use multiple operators, machines or any combination of people and machines (Aft 2000). It points out activity time, idle time, cycle time and utilization ratio of MS and machine. Therefore, this chart is used to establish man-machine ratio and to minimize idle MS/machine time, to optimize resources of MS and machines. However, as the number of machines operated by MS increases, it is more difficult to use the man-machine chart to comprehend the system complexity to fine tune manning ratio to improve MS or machine utilization (Aft 2000).

Simulation has proved effective in helping to sort through the complex issues surrounding manufacturing decision (Harrell, Ghosh, and Bowden 2003). Its ability to imitate a dynamic system make it ideally suited for manmachine ratio modeling. 


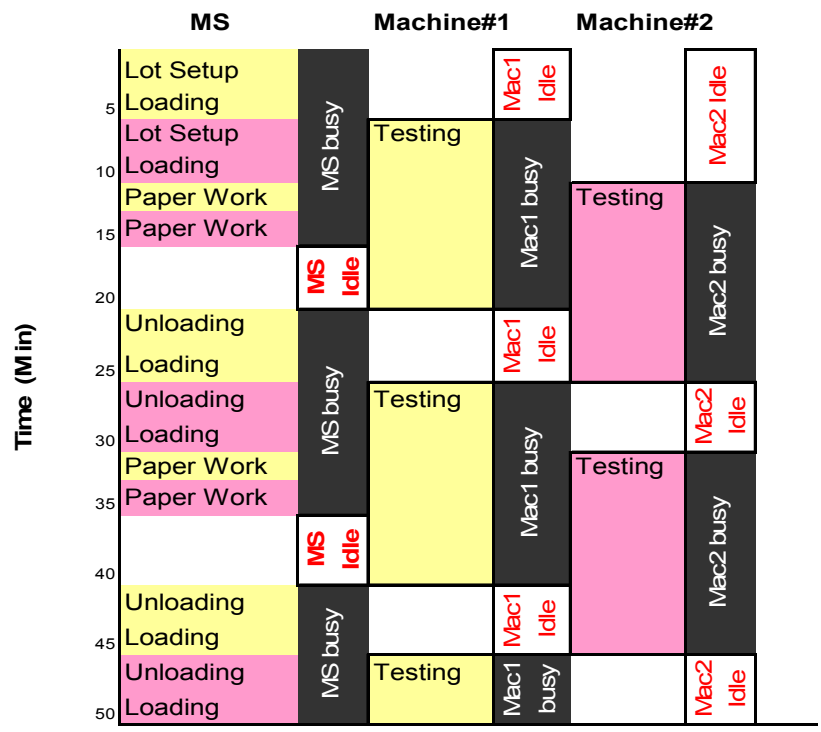

Figure 1: Man-machine chart

\section{SIMULATION EXPERIMENTS}

Man-machine ratio models can be built using discrete event simulation software, ProModel. This simulation models work elements of MS and equipment behavior to determine the optimal man-machine ratio. Typical events in man-machine simulation model are MS and machine work elements; as shown in Figure 2. Both machines and MS are modeled as resources. The model uses entities to simulate product lots that flow through manufacturing.

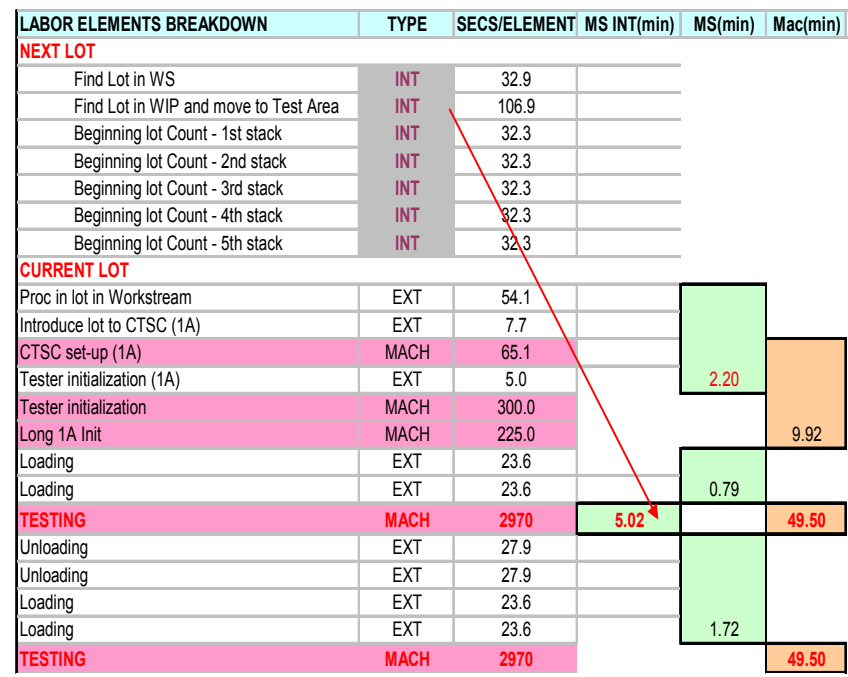

Figure 2: MS and machine work elements

In reality, MS resources are occupied to perform a sequence of work elements. Thus as illustrated in Figure 2, a logical sequence of MS work elements are grouped together in cyan boxes. Logic statement GET and WAIT are issued in simulation model to utilize MS resource for the duration of time to complete the group of work elements.
After which, MS resource will be freed using logic statement FREE. The 'free' MS waits for the next group of work elements that request for his/her resource again, which could be from the same machine or another machine. The same logic applies for machine utilization with the exception that machine resources will not be requested by other machines other than itself.

Simulation model has the capability to model internal work elements as how they happen in real life, an advantage over spreadsheet calculation. As illustrated in Figure 2, a logical group of internal work elements is modeled to be performed by MS when the machine is running. The example illustrates that setup activities of the next lot is executed by MS while machine is testing current lot. In the event that these lot setup activities can no longer be "internal" work elements when one MS attends to multiple machines, simulation can accurately model the activities as "external" work elements and factor in resulted machine idling time. As such, simulation render better accuracy of MS and machines resource utilization at different manning ratio. It is therefore a better tool to establish optimal manning ratio with comparison to spreadsheet calculation.

Initial prototype modeled the manning ratio being practiced in the production prior to improvement study. Machine throughput of simulation prototype was verified against runrate determined by Industrial Engineer to test if model was developed accurately. Once verified, model was expanded to include different manning ratio. Model validation is then performed on model's machine throughput against actual production output. The verification and validation process involve extensive data analysis to close and/or explain the gaps among these 3 elements of simulation throughput, IE runrate and actual production output.

Studies were performed on constraint tool, test machine for manning ratio from 1:1 to 1:4.

\section{SIMULATION RESULTS}

Figure 3 shows utilization of MS and test machine. The first and second bars from the top in green shows utilization of MS and test machine at manning ratio 1:1. The subsequent bars indicates utilization of MS and test machine at $1: 2,1: 3$ and 1:4 manning ratio respectively. Blue bars indicate idle time for either MS or machine. The utilization of test machine decreases as manning ratio increases. As manning ratio increases, MS resource utilization also increases with MS spending some time traveling to and fro machines as shown in yellow bars. As illustrated, simulation can account for activity time, idle time, cycle time and utilization ratio of MS and machine even when one MS tends to many machines. This is an advantage of simulation over man-machine chart. 


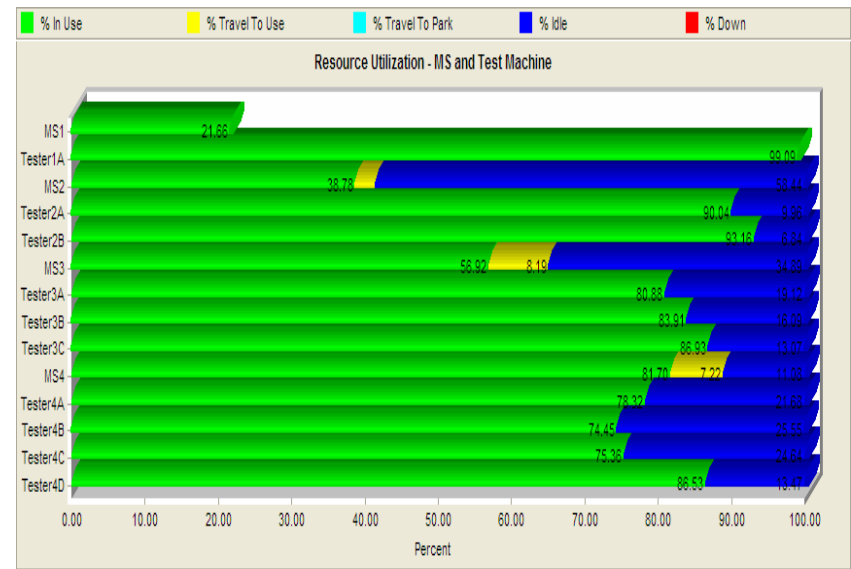

Figure 3: Resource utilization - MS and test machine

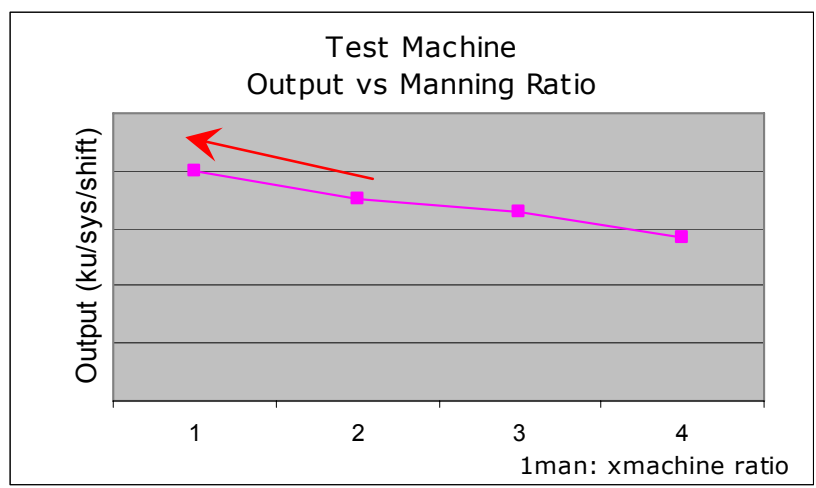

Figure 4: Output of tester at different manning ratio constraint tool - test machine

As shown in Figure 4, the output per test machine in a shift increases as MS operates less number of machines. A polynomial expression can be established between throughput and manning ratio. With this expression, impact to machine throughput by other higher manning ratios such as 1:5 and 1:6; can be identified without further simulation studies.

Management can make more informed decision on manning ratio based on the above simulation outputs; if headcount to be saved at acceptable degradation of tester output, or if more MS resources to be added to harvest better output from test machine.

The idea was then to conduct combination studies of constraint tools and non-constraint tools; to explore possibilities to reduce headcount at non-constraint tools at acceptable degradation of machine throughput, while transferring of these headcount from non-constraint tools to constraint tools to boost the output at constraint area, and ultimately the overall factory output. This is the implementation of the $2^{\text {nd }}$ and $3^{\text {rd }}$ steps of the five focusing steps of Theory of Constraint; namely decide how to exploit the system's constraint and subordinate everything else to the above decision. (Goldratt 1999)

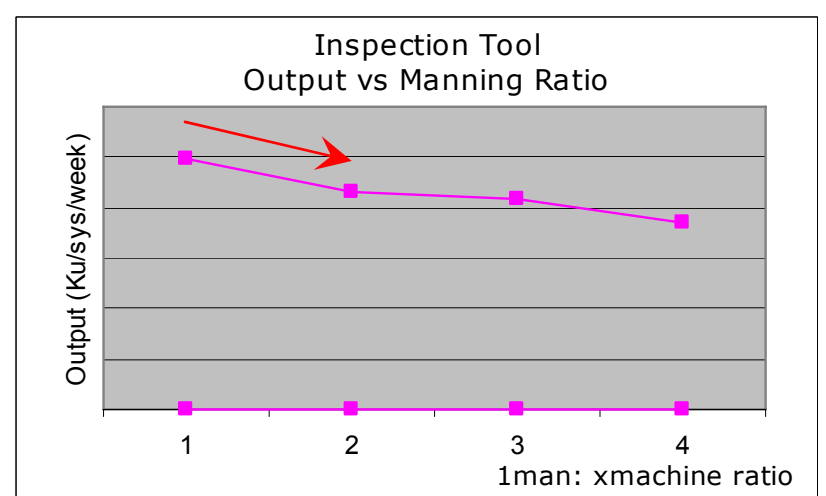

Figure 5: Output of inspection tool at different manning ratio

Non-constraint tool - inspection tool

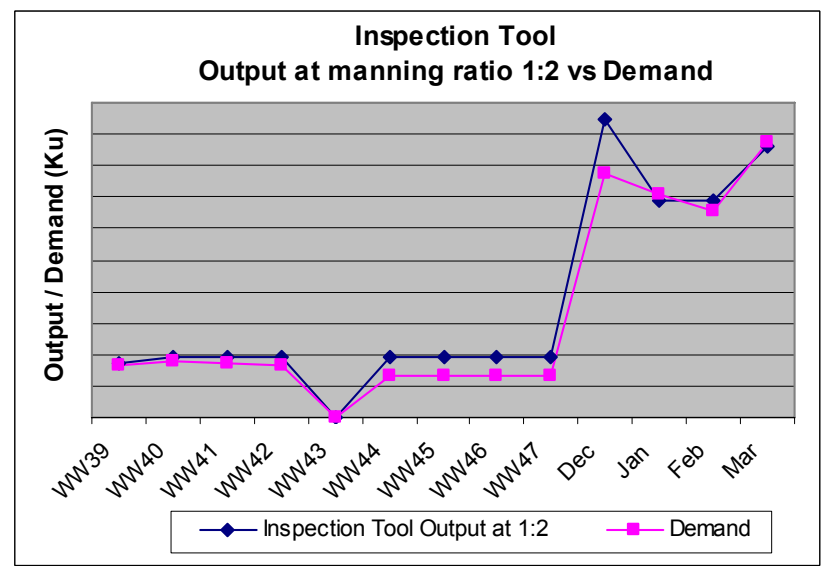

Figure 6 : Output of inspection tool vs demand for chipset product

As shown in Figure 4, 5 and 6 the simulation models demonstrate that by increasing manning ratio from existing 1:2 to $1: 1$ of constraint tool, test machine, will achieve more than $14 \%$ throughput improvements. Meanwhile, by modeling the non-constraint, inspection tool, we also learnt that by extending inspection tool manning ratio from 1:1 to $1: 2$, the productivity degradation of inspection tool is still able to support the build plan. Hence, results of our study facilitates decision made to optimally move headcount from non-constraint to constraint locations. 


\section{SUMMARY AND CONCLUSION}

In this paper, we discussed advantages of simulation over traditional methods, namely spreadsheet analysis and manmachine chart. We presented case studies which establish man-machine ratio using simulation at constraint and nonconstraint tool. It has been shown that simulation output can be used to facilitate decision making on headcount movement from non-constraint to constraint operation. Constraint tools can be "exploited" to produce optimal output with more headcounts released from non-constraint tool while non-constraint tool can retain to support demands at reduced outputs with remaining headcounts.

\section{REFERENCES}

Aft, L. S. 2000. Work measurement and methods improvement (Engineering design and automation). 3rd ed. 69-79. New York: Wiley Series in Engineering Design and Automation.

Institute of Industrial Engineers. 2007. Work design and measurement. Atlanta: Institute of Industrial Engineers

Harrell, C., B. K. Ghosh, and Bowden, R.O. 2003. Simulation using ProModel. 3rd ed. New York: McGraw-Hill, Inc.

Goldratt, E. M. 1999. Theory of constraints. Great Barrington: North River Press.

\section{AUTHOR BIOGRAPHIES}

HOAY HOON ONG is a member of the Analytic Solutions Development group at Intel, in Malaysia. She received her M.S. in Information Technology in Business from University of Humberside and Lincolnshire, UK. Hoay Hoon can be reached via email at

<hoay.hoon.ong@intel.com> 